\title{
364.
}

\section{ON A THEOREM RELATING TO FIVE POINTS IN A PLANE.}

[From the Philosophical Magazine, vol. xxix. (1865), pp. 460-464.]

Two triangles, $A B C, A^{\prime} B^{\prime} C^{\prime \prime}$ which are such that the lines $A A^{\prime}, B B^{\prime}, C C^{\prime}$ meet in a point, are said to be in perspective; and a triangle $A^{\prime} B^{\prime} C^{\prime}$, the angles $A^{\prime}, B^{\prime}, C^{\prime}$ of which lie in the sides $B C, C A, A B$ respectively, is said to be inscribed in the triangle $A B C$; hence, if $A^{\prime}, B^{\prime}, C^{\prime}$ are the intersections of the sides by the lines $A O, B O, C O$ respectively (where $O$ is any point whatever), the triangle $A^{\prime} B^{\prime} C^{\prime}$ is said to be perspectively inscribed in the triangle $A B C$, viz. it is so inscribed by means of the point $O$.

We have the following theorem, relating to any triangle $A B C$, and two points $O, O^{\prime}$. If in the triangle $A B C$, by means of the point $O$, we inscribe a triangle $A^{\prime} B^{\prime} C^{\prime}$, and in the triangle $A^{\prime} B^{\prime} C^{\prime}$, by means of the point $O^{\prime}$, we inscribe a triangle $\alpha \beta \gamma$, then the triangles $A B C, \alpha \beta \gamma$ are in perspective, viz. the lines $A \alpha, B \beta, C \gamma$ will meet in a point.

This is very easily proved analytically; in fact, taking $x=0, y=0, z=0$ for the equations of the lines $B^{\prime} C^{\prime}, C^{\prime} A^{\prime}, A^{\prime} B^{\prime}$ respectively, and $(X, Y, Z)$ for the coordinates of the point $O$, then the coordinates of $(A, B, C)$ are found to be $(-X, Y, Z),(X,-Y, Z)$, $(X, Y,-Z)$ respectively. Moreover, if $\left(X^{\prime}, Y^{\prime}, Z^{\prime}\right)$ are the coordinates of the point $O^{\prime}$, then the coordinates of $(\alpha, \beta, \gamma)$ are found to be

$$
\left(0, Y^{\prime}, Z^{\prime}\right),\left(X^{\prime}, 0, Y\right),\left(X^{\prime}, Y^{\prime}, 0\right)
$$

respectively. Hence the equations of the lines $A \alpha, B \beta, C \gamma$ are respectively

$$
\left|\begin{array}{rrr}
x, & y, & z \\
-X, & Y, & Z \\
0, & Y^{\prime}, & Z^{\prime}
\end{array}\right|=0,\left|\begin{array}{rrr}
x, & y, & z \\
X, & -Y, & Z \\
X^{\prime}, & 0, & Z^{\prime}
\end{array}\right|=0,\left|\begin{array}{rrr}
x, & y, & z \\
X, & Y, & -Z \\
X^{\prime}, & Y^{\prime}, & 0
\end{array}\right|=0
$$


that is

$$
\begin{aligned}
& x\left(Y^{\prime}-Y^{\prime} Z\right)+y\left(\quad Z^{\prime} X\right)+z\left(-X Y^{\prime} \quad\right)=0, \\
& x\left(-Y Z^{\prime}\right)+y\left(Z X^{\prime}-Z^{\prime} X\right)+z\left(\quad X^{\prime} Y\right)=0 \text {, } \\
& x\left(+Y^{\prime} Z\right)+y\left(-Z X^{\prime} \quad\right)+z\left(X Y^{\prime}-X^{\prime} Y\right)=0,
\end{aligned}
$$

which are obviously the equations of three lines which meet in a point.

But the theorem may be exhibited as a theorem relating to a quadrangle 1234 and a point $O^{\prime}$; for writing $1,2,3,4$ in place of $A, B, C, O$, the triangle $A^{\prime} B^{\prime} C^{\prime}$ is in fact the triangle formed by the three centres $41.23,42.31,43.12$ of the quadrangle 1234, hence the triangle in question must be similarly related to each of the four triangles $423,431,412,123$; or, forming the diagram

\begin{tabular}{l|l|l|l|l} 
& $P$ & $Q$ & $R$ & $S$ \\
41.23 & 4 & 3 & 2 & 1 \\
42.31 & 3 & 4 & 1 & 2 \\
43.12 & 2 & 1 & 4 & 3
\end{tabular}

we have the following form of the theorem: viz. the lines

$$
\begin{aligned}
& \alpha 4, \beta 3, \gamma^{2} \text { meet in a point } P \text {, } \\
& \alpha 3, \beta 4, \gamma 1 \quad, \quad, Q \text {, } \\
& \alpha 2, \beta 1, \gamma 4 \quad \text { " \# } R \text {, } \\
& \alpha 1, \beta 2, \gamma 3 \quad " \quad, S \text {, }
\end{aligned}
$$

or, what is the same thing, we have with the points $1,2,3,4$ and the point $O^{\prime}$ constructed the four points $P, Q, R, S$ such that

$$
\begin{aligned}
& 1 S, 2 R, 3 Q, 4 P \text { meet in a point } \alpha \text {, } \\
& 2 S, 1 R, 4 Q, 3 \beta \quad \text { " " } \beta \text {, } \\
& 3 S, 4 R, 1 Q, 2 P \quad " \quad " \gamma \text {. }
\end{aligned}
$$

The eight points $1,2,3,4, P, Q, R, S$ form a figure such as the perspective representation of a parallelopiped, or, if we please, a cube; and not only so, but the

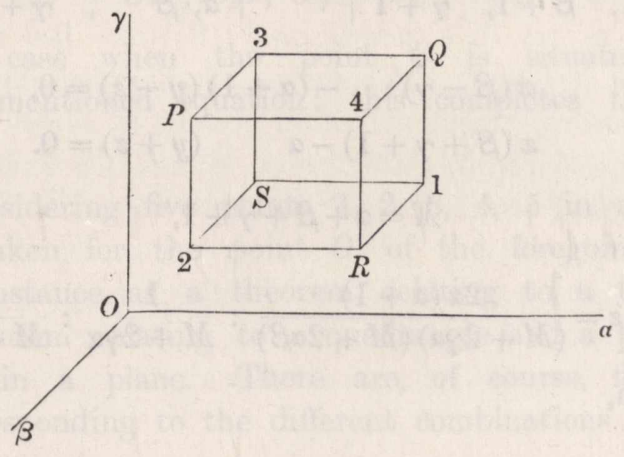

plane figure is really a certain perspective representation of the cube; this identification depends on the following two theorems:

c. v. 
1. Considering the four summits $1,2,3,4$, which are such that no two of them belong to the same edge, then, if through any point $O$ we draw

the line $O A^{\prime}$ meeting the lines 41,23 ,

$$
\begin{array}{lllll}
\prime & O B^{\prime} & & & \\
\prime & O C^{\prime} & & & 42,31,
\end{array}
$$

and the lines $O \alpha, O \beta, O \gamma$ parallel to the three edges of the cube respectively, the three planes $\left(O A^{\prime}, O \alpha\right),\left(O B^{\prime}, O \beta\right),\left(O C^{\prime}, O \gamma\right)$ will meet in a line.

2. For a properly selected position of the point $O$,

the lines $O B^{\prime}, O C^{\prime}, \quad O \alpha$ will lie in a plane,

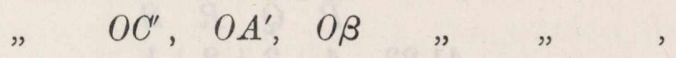

$$
\begin{aligned}
& \text { " } O A^{\prime}, O B^{\prime}, \quad O \gamma
\end{aligned}
$$

In fact for such a position of $O$, projecting the whole figure on any plane whatever, the lines $01,02,03,04, O P, O Q, O R, O S, O \alpha, O \beta, O \gamma, O A^{\prime}, O B^{\prime}, O C^{\prime}$ meet the plane of projection in the points $1,2,3,4, P, Q, R, S, \alpha, \beta, \gamma, A^{\prime}, B^{\prime}, C^{\prime}$ related to each other as in the last-mentioned form of the plane theorem. To prove the two solid theorems, take $O$ for the origin, $O \alpha, O \beta, O \gamma$ for the axes, $(\alpha, \beta, \gamma)$ for the coordinates of the summit $S$, and 1 for the edge of the cube,

$$
\begin{aligned}
& \text { the coordinates of } 1 \text { are } \alpha+1, \beta, \quad \gamma, \\
& \qquad \begin{array}{ll}
2, \alpha, \quad \beta+1, \gamma, \\
" & 3, \alpha, \quad \beta, \quad \gamma+1, \\
" & 4, \alpha+1, \beta+1, \gamma+1 .
\end{array}
\end{aligned}
$$

The equations of the line $O A^{\prime}$, or say of the line $O(41,23)$, are those of the planes 041,023 , viz. these are

that is

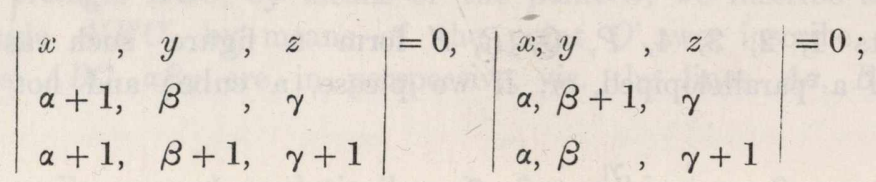

Writing for shortness

$$
\begin{array}{ll}
x(\beta-\gamma)-(\alpha+1) & (y-z)=0, \\
x(\beta+\gamma+1)-\alpha & (y+z)=0 .
\end{array}
$$

these equations give

$$
M=\alpha+\beta+\gamma+1
$$

$$
x: y: z=\frac{2 \alpha(\alpha+1)}{(M+2 \gamma \alpha)(M+2 \alpha \beta)}: \frac{1}{M+2 \gamma \alpha}: \frac{1}{M+2 \alpha \beta} ;
$$

or, completing the system,

for line $O A^{\prime}$ we have

$$
x: y: z=\frac{2 \alpha(\alpha+1)}{(M+2 \gamma \alpha)(M+2 \alpha \beta)}: \frac{1}{M+2 \gamma \alpha}: \frac{1}{M+2 \alpha \beta}
$$


for line $O B^{\prime}$ we have

for line $O C^{\prime \prime}$ we have

$$
x: y: z=\frac{1}{M+2 \beta \gamma}: \frac{2 \beta(\beta+1)}{(M+2 \alpha \beta)(M+2 \beta \gamma)}: \frac{1}{M+2 \alpha \beta} ;
$$

$$
x: y: z=\frac{1}{M+2 \beta \gamma}: \frac{1}{M+2 \gamma \alpha}: \frac{2 \gamma(\gamma+1)}{(M+2 \beta \gamma)(M+2 \gamma \alpha)} .
$$

The equations of the lines $O \alpha, O \beta, O \gamma$ are of course $(y=0, z=0),(z=0, x=0)$, $(x=0, y=0)$ respectively; and we therefore see at once that the planes $\left(O A^{\prime}, O \alpha\right)$, $\left(O B^{\prime}, O \beta\right),\left(O C^{\prime}, O \gamma\right)$ meet in a line, viz. in the line which has for its equations

$$
x: y: z=\frac{1}{M+2 \beta \gamma}: \frac{1}{M+2 \gamma \alpha}: \frac{1}{M+2 \alpha \beta} .
$$

The lines $O B^{\prime}, O C^{\prime}, O \alpha$ will lie in a plane, if only

that is

$$
1=\frac{4 \beta \gamma(\beta+1)(\gamma+1)}{(M+2 \beta \gamma)^{2}}
$$

$$
(M+2 \beta \gamma)^{2}=4 \beta \gamma(\beta+1)(\gamma+1)
$$

or, as this may be written,

that is

$$
M^{2}+4 \beta \gamma(\alpha+\beta+\gamma+1+\beta \gamma)=4 \beta \gamma(\beta \gamma+\beta+\gamma+1)
$$

or, what is the same thing,

$$
M^{2}+4 \alpha \beta \gamma=0,
$$

$$
(\alpha+\beta+\gamma+1)^{2}+4 \alpha \beta \gamma=0 ;
$$

and from the symmetry of this equation we see that, when it is satisfied,

the lines $O B^{\prime}, O C^{\prime}, O \alpha$ will lie in a plane,

$$
\begin{aligned}
& O C^{\prime}, O A^{\prime}, O \beta \\
& O A^{\prime}, O B^{\prime}, O \gamma
\end{aligned}
$$

viz. this will be the case when the point $O$ is situate in the cubic surface represented by the last-mentioned equation; this completes the demonstration of the solid theorems.

It is clear that considering five points $1,2,3,4,5$ in a plane, then, since any one of these may be taken for the point $O^{\prime}$ of the foregoing theorem, the theorem exhibited in the first instance as a theorem relating to a triangle and two points, and afterwards as a theorem relating to a quadrangle and a point, is really a theorem relating to five points in a plane. There are, of course, five different systems of points $(P, Q, R, S)$, corresponding to the different combinations of four out of the five points.

Cambridge, March 6, 1865. 